\title{
Improving bioorthogonal protein ubiquitylation by click reaction
}

\author{
Daniel Schneider ${ }^{\dagger}$, Tatjana Schneider ${ }^{\dagger}$, Daniel Rösner ${ }^{\dagger}$, Martin Scheffner*, Andreas Marx* \\ Department of Chemistry, Konstanz Research School Chemical Biology, University of Konstanz, 78457 Konstanz, Germany \\ Department of Biology, Konstanz Research School Chemical Biology, University of Konstanz, 78457 Konstanz, Germany
}

Keywords:

Ubiquitin

Unnatural amino acids

Click reaction

DNA polymerase $\beta$

Protein conjugation

\begin{abstract}
A B S T R A C T
Posttranslational modification of proteins with ubiquitin (ubiquitylation) regulates numerous cellular processes. Besides functioning as a signal for proteasomal degradation, ubiquitylation has also non-proteolytic functions by altering the biochemical properties of the modified protein. To investigate the effect(s) of ubiquitylation on the properties of a protein, sufficient amounts of homogenously and well-defined ubiquitylated proteins are required. Here, we report on the elaboration of a method for the generation of high amounts of site-specifically mono-ubiquitylated proteins. Firstly, a one-step affinity purification scheme was developed for ubiquitin containing the unnatural amino acid azidohomoalanine at the C-terminal position. This ubiquitin was conjugated in a click reaction to recombinant DNA polymerase $\beta$, equipped with an alkyne function at a distinct position. Secondly, addition of defined amounts of SDS to the reaction significantly improved product formation. With these two technical improvements, we have developed a straight forward procedure for the efficient generation of site-specifically ubiquitylated proteins that can be used to study the effect(s) of ubiquitylation on the activities/ properties of a protein.
\end{abstract}

\section{Introduction}

Posttranslational modifications of proteins play an important role in regulating cellular activities by enabling cells to respond to intra- and extracellular stimuli in a directed and dynamic manner. ${ }^{1}$ A prominent modification that affects many, if not all, proteins in a eukaryotic cell is the covalent attachment of ubiquitin ('ubiquitylation') that plays a key role in the control of signaling pathways and other fundamental cellular processes. ${ }^{2-5}$

Ubiquitin (Ub) is an essential eukaryotic protein consisting of 76 amino acids and is highly conserved in evolution (e.g., Ub derived from baker's yeast and humans share $96 \%$ sequence identity). Modification of proteins with Ub can result in the formation of different conjugates. In its simplest form, termed mono-ubiquitylation, a single moiety of $\mathrm{Ub}$ is attached to a Lys residue of a substrate. The linkage is formed via an isopeptide bond between the $\varepsilon$-amino group of the Lys residue and the carboxyl group of the C-terminal Gly (G76) of Ub. Alternatively, several Ub moieties can be attached to different Lys residues of the substrate, which is referred to as multiple mono-ubiquitylation. Furthermore, Ub itself contains seven Lys and as each of these as well as the N-terminal amino group can serve as acceptor for Ub, proteins can be modified

\footnotetext{
* Corresponding authors. Tel.: +49 753188 5139; fax: +497531 885140.

E-mail addresses: martin.scheffner@uni-konstanz.de (M. Scheffner), andreas. marx@uni-konstanz.de (A. Marx).

$\uparrow$ These authors contributed equally to this work
}

by Ub chains that differ in length and the Lys residue(s) used for chain formation ('poly-ubiquitylation'). ${ }^{6}$ It is commonly assumed that the type of ubiquitylation determines the fate of a modified protein. ${ }^{7}$ The best known function of poly-ubiquitylation is targeting proteins for proteasomal degradation. However, poly-ubiquitylation and in particular mono-ubiquitylation have many other, non-proteolytic functions. $^{8-10}$ For example, mono-ubiquitylation can alter protein-protein interaction properties, ${ }^{11-13}$ sub-cellular localization $^{14,15}$ or the biochemical function ${ }^{16,17}$ of a protein. Along these lines, mono-ubiquitylation plays an important role in the regulation of proteins involved in DNA repair pathways including base excision repair. ${ }^{18-22}$ Nevertheless, in many cases it remains obscure how Ub modification affects the activity of the respective proteins.

A general obstacle in the ubiquitin research field has been the lack of sufficient amounts of homogenously mono-ubiquitylated proteins (i.e., modified at a defined Lys residue) for detailed biochemical analyses. ${ }^{23-25}$ Recently, a number of approaches have been developed for site-specific conjugation of $\mathrm{Ub}$ to $\mathrm{Ub}$ or other proteins, ${ }^{26-28}$ including native chemical ligation, ${ }^{29-31}$ oxime-based ligation, ${ }^{32}$ thioether ligation, ${ }^{33}$ intein based two-step expressed protein ligation (EPL $)^{34}$ and $\mathrm{Cu}(\mathrm{I})$-catalyzed azide-alkyne cycloaddition (click reaction). ${ }^{35-38}$ Taking advantage of the click reaction, our group established a straightforward approach that combines chemical and biological tools without the need of solid phase peptide synthesis or laborious eukaryotic cell culture. ${ }^{36,39,40}$

The click reaction relies on a 1,3-dipolar cycloaddition between an azide and a terminal alkyne that results in the formation of 
stable 1,4-disubstituted triazoles at physiological conditions via $\mathrm{Cu}(\mathrm{I})$ catalysis. ${ }^{41-43}$ For the use with proteins, the method has been refined over the years. Instead of using $\mathrm{Cu}(\mathrm{I})$ salts, the catalyst can be prepared in situ by reduction of $\mathrm{Cu}(\mathrm{II})$ salts like $\mathrm{CuSO}_{4}{ }^{44}$ Besides ascorbate, the water soluble tris-(carboxyethyl)phosphine (TCEP) was shown to be a suitable reductant when working with proteins. ${ }^{45}$ Furthermore, the instability of $\mathrm{Cu}(\mathrm{I})$ favors oxidation to $\mathrm{Cu}(\mathrm{II})$ or disproportionation to $\mathrm{Cu}(0)$ and $\mathrm{Cu}(\mathrm{II})$ shutting down the $\mathrm{Cu}(\mathrm{I})$-mediated catalytic cycle, for example, in the presence of atmospheric oxygen. Therefore, using click reaction for the modification of proteins under aerobic conditions requires additives that stabilize the $\mathrm{Cu}(\mathrm{I})$ species and prevent oxidative damage of proteins. Polytriazoles such as tris-(benzyltriazolylmethyl)amine (TBTA) or tris-(hydroxypropyltriazolylmethyl)amine (THPTA) have been identified as powerful $\mathrm{Cu}(\mathrm{I})$-stabilizing ligands while enhancing the catalytic activity of $\mathrm{Cu}(\mathrm{I})$ and thereby accelerating the reaction. ${ }^{46}$

To install the bioorthogonal functionalities enabling click reactions with ubiquitin, we make use of unnatural amino acids (UAAs). Their incorporation into proteins is enabled by the fact that analogs of naturally occurring amino acids (AAs) are often accepted by the aminoacyl tRNA synthetase (AARS)/tRNA ${ }^{\text {AA }}$ pair of the natural AA. Particularly, the methionine analog azidohomoalanine (Aha, Fig. 1A) and the propargyl-protected pyrrolysine (Pyl) analog (Plk, Fig. 1B) have been used. The resulting non-natural triazole bond provides several advantages. It mimics the peptide bond by offering similar electronic properties and configuration of atoms, ${ }^{41,47-49}$ while resisting proteolytic cleavage.

To provide a C-terminal azide, the Gly76 codon of Ub was mutated to a Met codon. The resulting Ub variant (Ub G76M) was expressed in Escherichia coli and incorporation of the Met analog Aha was achieved using the selective pressure incorporation method (SPI). ${ }^{36}$ SPI utilizes bacterial strains that are auxotrophic for one of the twenty canonical $\mathrm{AA}{ }^{50}$ By exploiting the inability of the AARS to distinguish between structurally and chemically similar substrates $^{51}$, replacement of the canonical AA with its analog in the whole proteome is achieved. ${ }^{52}$ As Ub G76M contains two Met/Aha residues (encoded by the initial ATG codon and the ATG codon introduced at position 76), the initial Aha residue needs to be removed to ensure that click reaction occurs exclusively at position 76. This was achieved by co-translational removal of the $\mathrm{N}$ terminal Aha by the endogenous Met aminopeptidase (MetAP). ${ }^{36}$ However, this approach may not be feasible for Met analogs other than Aha, as they may not or only poorly be recognized by MetAP. To introduce the alkyne functionality, we used the propargylderivatized pyrrolysine (Pyl) analog Plk in combination with the PylRS/tRNA ${ }^{\text {Pyl }}$ pair of Methanosarcina barkeri. Upon introduction into $E$. coli, this orthogonal pair enables the suppression of an amber stop codon (UAG) when a suitable (U)AA, like Plk, is provided. $^{53-55}$ The method is referred to as amber codon suppression (stop codon suppression, SCS) and was successfully
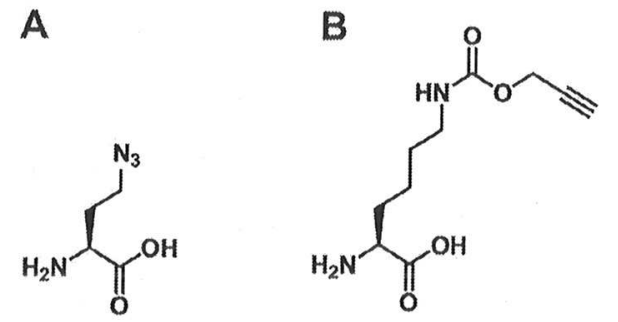

Azidohomoalanine (Aha)

Propargyl-derivatized lysine (PIk)

Figure 1. Chemical structures of the unnatural amino acids incorporated. used by us for the replacement of a Lys residue by Plk at defined positions of Ub and PCNA. ${ }^{36,39}$

Human DNA polymerase ( $\mathrm{Pol}) \beta$, which plays an important role in base excision repair, ${ }^{56-58}$ is a known target for ubiquitylation with three Lys residues (Lys41, Lys61, and Lys81) serving as acceptor sites for Ub. ${ }^{18.57}$ However, if and how mono-ubiquitylation affects the biochemical properties of Pol $\beta$ is currently unknown. As a first step to address this important question, we developed a one-step purification protocol for Ub variants containing Aha at position 76 that is also suitable for any other Met analog. Furthermore, while improving the efficiency of the click reaction between Ub G76Aha and Plk-containing Pol $\beta$, we found that in the presence of the detergent sodium dodecyl sulfate (SDS), yields up to $70 \%$ of Pol $\beta$-Ub conjugates were achieved.

\section{Results and discussion}

For the generation of defined Ub conjugates by click reaction, the Met analog incorporated at position 1 of Ub (note that Ub does not contain any other Met) by SPI needs to be co- or posttranslationally removed. While in principle the removal can be achieved during expression of $\mathrm{Ub}$ in bacteria by MetAP, this enzymatic reaction may not be quantitative. Indeed, we found that cleavage efficiency of the initial Aha varied between different preparations and, moreover, that removal of the Met analog homopropargylglycine (Hpg) was generally rather inefficient (data not shown).

To develop an SPI-based approach that is generally applicable regardless of the Met analog used, we reasoned that proteolytic removal of the first AA of Ub or a Ub fusion protein should be performed under defined in vitro conditions by using site-specific proteases. Hence, we introduced different protease cleavage sites between the translational start codon and the second codon of Ub. Additionally, to allow easy separation of cleaved Ub from unprocessed $\mathrm{Ub}$, an affinity tag was inserted upstream of the protease cleavage site. Initially, Ub variants with an $\mathrm{N}$-terminal $\mathrm{His}_{6}$-tag followed by a thrombin or TEV protease cleavage site were generated. However, with both constructs hardly any cleavage was observed, even when different linkers between the $\mathrm{His}_{6}$-tag and the cleavage sites were tested (data not shown). Thus, in the next set of experiments, another affinity tag was used. An N-terminal fusion of glutathione S-transferase (GST) to Ub has previously been used for affinity purification. Furthermore, a thrombin cleavage site between GST and Ub has been shown to permit quantitative cleavage of the fusion protein. ${ }^{59}$ Accordingly, a cDNA of Ub without the initial ATG codon but encoding the G76M mutation was cloned into the pGEX2TK vector downstream of GST and the thrombin cleavage site. Following expression of the fusion construct in the presence of Aha, purification of Ub G76Aha was readily achieved by a one-step approach (Fig. 2A).

In detail, upon expression, cell harvest and lysis, the clarified cell lysate was loaded onto a GST trap column, followed by washing with $1 \times$ PBS buffer until elution of proteins was no longer observed, as confirmed by SDS-PAGE (Fig. 2B, lanes 1-3). Then, thrombin cleavage was carried out with $20 \mathrm{U}$ overnight at room temperature. Finally, Ub G76Aha was eluted by washing with $1 \times$ PBS buffer (Fig. 2B, lanes 4-8). The marginal impurities visible at higher molecular weights were avoided by decreasing the amount of thrombin used to $5 \mathrm{U}$ (Fig. 3A). The identity of Ub G76Aha and the absence of the N-terminal GST was verified by mass spectrometry (Fig. 3A).

Remarkably, replacement of the eight Met residues present in GST by Aha does apparently not affect its stability and affinity for glutathione in a significant manner. The recombinant GST is still expressed in the soluble fraction and binds to glutathion (Fig. 2B, 


\section{A Growth of E.coli B834 with pGEX2TK_Ubiquitin G76M} in NMM medium with methionine overnight

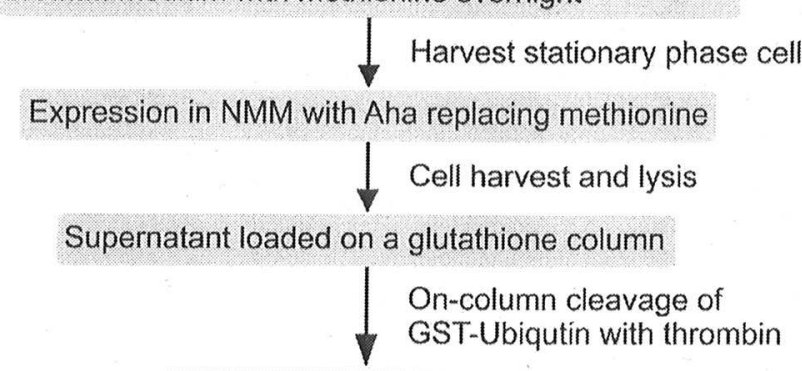

\section{Purified Ubiquitin c76Aha}
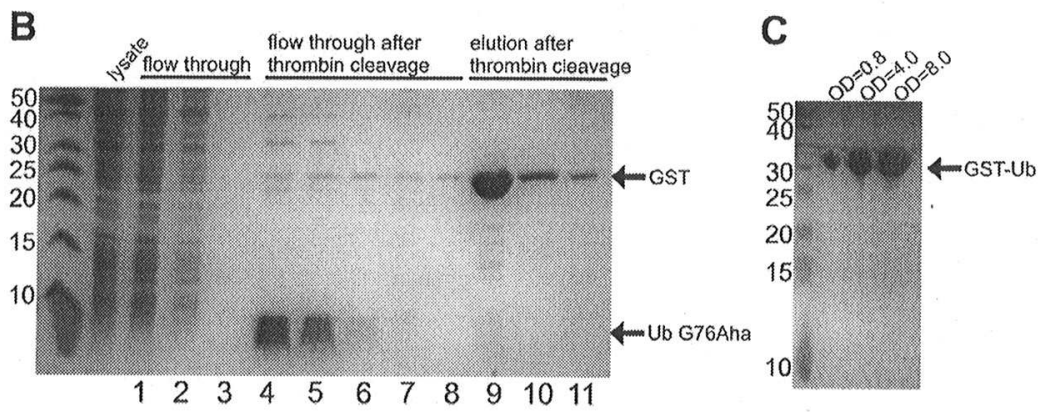

Figure 2. Expression of Ub as GST-fusion protein and purification of Ub G76Aha. (A) Schematic workflow of the approach developed; (B) analysis of the purification of Ub G76Aha. Aliquots of the cell lysate loaded onto the column (Lysate), flow through of the column (lanes 1-3), elution of Ub G76Aha after thrombin cleavage (lanes 4-8), and elution of GST with $10 \mathrm{mM}$ glutathione (lanes 9-11) were analyzed by SDS-PAGE followed by staining with coomassie blue; (C) effect of different cell densities of the culture at expression start on the yield of GST-Ub G76Aha fusion protein. For each sample the amount of protein bound to glutathione beads is shown.
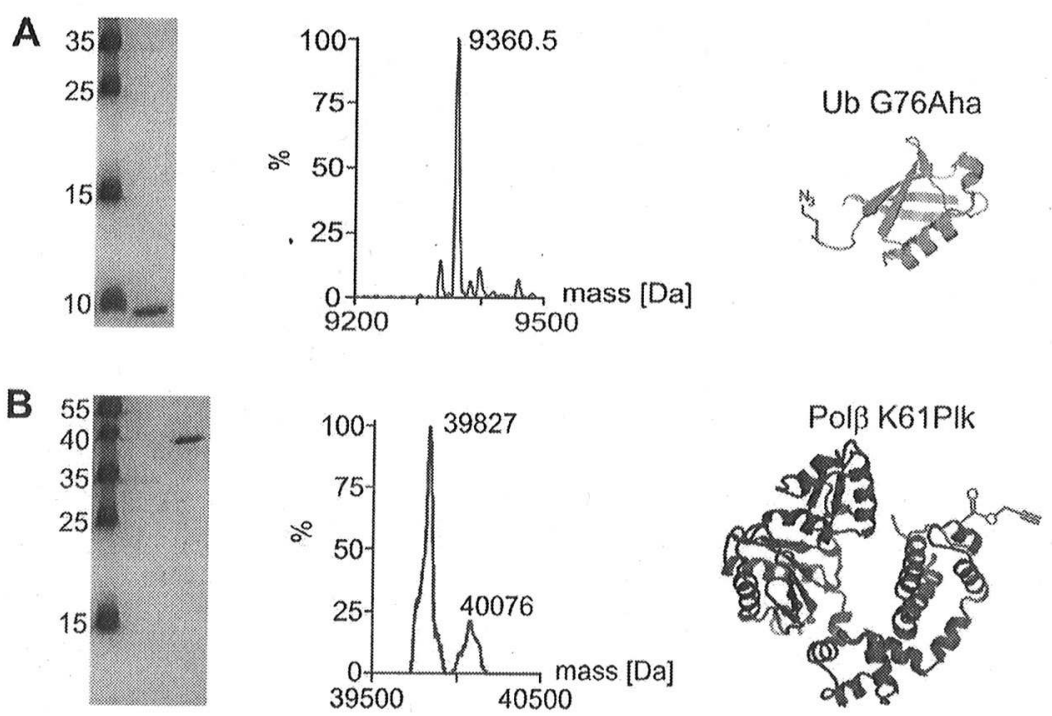

Figure 3. Incorporation of Aha and Plk. (A) Left: purified Ub G76Aha; middle: deconvoluted ESI-MS spectrum of thrombin-cleaved Ub G76Aha (calculated mass: 9630.6): right: scheme of Ub G76Aha; (B) left: purified Pol $\beta$ K61Plk; middle: deconvoluted ESI-MS spectrum of full-length Pol $\beta$ K61 Plk (calculated mass: 39,827 and 40,085 in case of $\alpha-\mathrm{N}$-6-phosphogluconolyation of $\mathrm{His}_{6}$-tag); right: scheme of Pol $\beta$ K61Plk.

lanes 9-11). However, GST-Ub G76Aha was expressed at significantly lower levels than untagged Ub G76Aha. To optimize this process, expression at different cell densities was tested (Fig. 2C), since in case of SCS, expression at elevated cell densities was reported to result in enhanced protein yield. ${ }^{60}$ Indeed, we also found for SPI that expression at an increased cell density greatly improves the yield of GST-Ub G76Aha (Fig. 2C). Normalized to the amount of free Aha added, the highest expression of the fusion construct was observed at an OD of 8 . Consequently, all further expressions were carried out under these conditions. Following this approach, Ub G76Aha was purified with a yield of approximately $9.5 \mathrm{mg} / \mathrm{l}$ expression culture. 
A

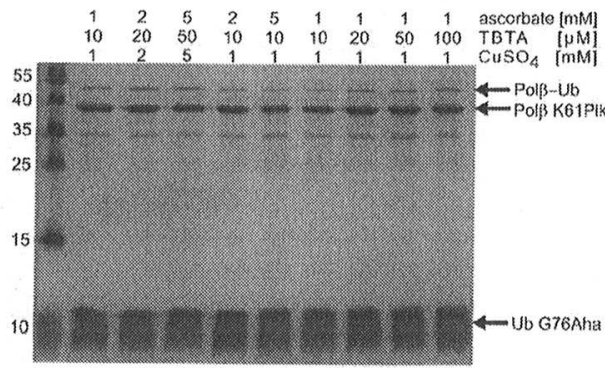

C

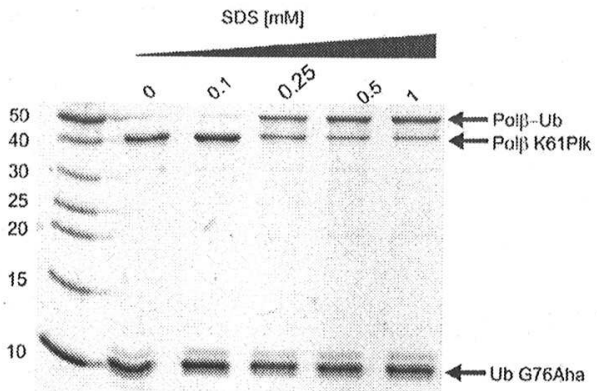

B

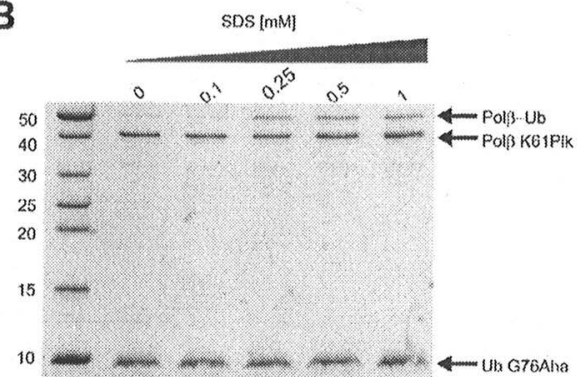

D

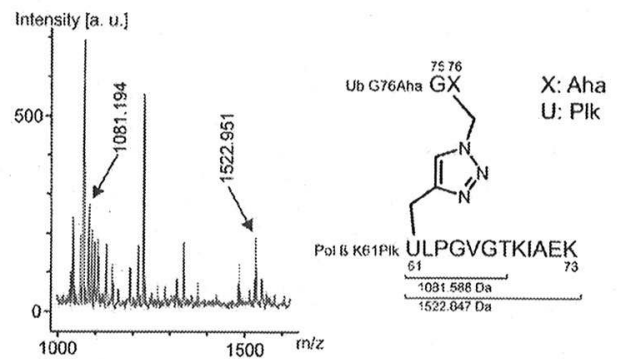

Figure 4. Mono-ubiquitylation of Pol $\beta$ by click reaction of Ub G76Aha and Pol $\beta$ K61Plk. (A) SDS-PAGE analysis of click reactions with ascorbate (1-5 mM), TBTA (10$100 \mu \mathrm{M}), \mathrm{CuSO}_{4}(1-5 \mathrm{mM})$ carried out for $2 \mathrm{~h}$ at $4^{\circ} \mathrm{C} ;(\mathrm{B})$ analysis of click reactions performed with $1 \mathrm{mM}$ ascorbate, $10 \mu \mathrm{M} \mathrm{TBTA}$ and $1 \mathrm{mM} \mathrm{CuSO} \mathrm{m}_{4}$ for $15 \mathrm{~min}$ at $4{ }^{\circ} \mathrm{C}$. Samples were supplemented with increasing amounts of SDS as indicated; (C) analysis of click reactions performed with $2 \mathrm{mM}$ TCEP, $20 \mu \mathrm{M}$ TBTA and $2 \mathrm{mM} \mathrm{CuSO}{ }_{4}$ for $2 \mathrm{~h}$ at $4{ }^{\circ} \mathrm{C}$. Samples were supplemented with increasing amounts of SDS as indicated; (D) left: MALDI-MS spectrum of trypsin-digested Pol $\beta$ K61Plk-Ub G76Aha (Pol $\beta$-Ub); observed masses of clicked fragments are indicated. Right: scheme of expected fragments including calculated mass.

In order to investigate the reactivity of purified Ub G76Aha (Fig. 3A), we performed a click reaction with Pol $\beta$, a known substrate of Ub. ${ }^{18.57}$ To this end, a human Pol $\beta$ variant with Plk incorporated at position 61 providing the alkyne functionality was generated. Plk was incorporated by SCS and Pol $\beta$ K61Plk was purified to homogeneity by a two-step approach (Fig. 3B). ESI-MS analysis of the purified protein revealed two species, both corresponding to the mass of the full-length protein with Plk incorporated instead of a Lys residue. Spontaneous $\alpha-N-6$-phosphogluconoylation of the $\mathrm{His}_{6}$-tag in E. coli is responsible for the observed deviation of $249 \mathrm{Da}^{61}$

Purified Ub G76Aha and Pol $\beta$ K61Plk were incubated under click reaction conditions. The molar excess of Ub G76Aha over Pol $\beta$ K61Plk varied from five-tenfold in order to maximize conversion of the latter protein to the conjugated form. Initially, reactions were carried out with ascorbate as reductant for $2 \mathrm{~h}$ at $4{ }^{\circ} \mathrm{C}$. Furthermore, the influence of different total concentrations and ratios of ascorbate: TBTA $\mathrm{CuSO}_{4}$ on the reaction efficiency was evaluated (Fig. 4A). In all cases tested, only minor conjugation of Pol $\beta$-Ub could be detected, as determined by the appearance of a new band on the SDS gel at about $50 \mathrm{kDa}$. Therefore, additional reaction parameters including $\mathrm{Cu}(\mathrm{I})$ sources, reductants, $\mathrm{Cu}(\mathrm{I})$ ligands, incubation time and temperature were varied. However, the reaction efficiency remained at a low level (data not shown). Finally, the effect of varying concentrations of SDS in the click reaction was analyzed (Fig. 4B and C). Concentrations of $0.25 \mathrm{mM}$ SDS or higher led to a significant increase in product formation with yields up to $40 \%$ (Fig. 4B). These reactions were performed with $1 \mathrm{mM}$ ascorbate, $10 \mu \mathrm{M}$ TBTA and $1 \mathrm{mM} \mathrm{CuSO}_{4}$ for $15 \mathrm{~min}$ at $4{ }^{\circ} \mathrm{C}$. To investigate if the effect of SDS is independent of parameters such as incubation time and reductant, click reactions were also performed with $2 \mathrm{mM}$ TCEP, $20 \mu \mathrm{M} \mathrm{TBTA}$ and $2 \mathrm{mM} \mathrm{CuSO}_{4}$ for $2 \mathrm{~h}$ at $4{ }^{\circ} \mathrm{C}$ (Fig. 4C). In this case, a product yield of up to $70 \%$ was observed with an SDS concentration of $0.25 \mathrm{mM}$ or higher.
For further analysis, the band corresponding to the Pol $\beta$-Ub conjugate was excised from the SDS gel and subjected to tryptic digest. Subsequently, the fragments were analyzed by mass spectrometry. The triazole linkage between the C-terminus of $\mathrm{Ub}$ and position 61 of Pol $\beta$ was identified by two individual fragments (Fig. 4D). This result verifies that the anticipated reaction product was formed and demonstrates the incorporation of the UAAs at the expected positions.

\section{Conclusion}

In this work, we have developed an efficient method for generation and purification of Ub variants containing the Met analog Aha at the $\mathrm{C}$-terminus. Furthermore, utilizing the $\mathrm{Cu}(\mathrm{I})$-catalyzed azide-alkyne reaction, we generated homogenously mono-ubiquitylated Pol $\beta$ in vitro. During these studies, we found that the addition of SDS at concentrations that presumably do not distort the conformation of a protein $^{62,63}$ greatly enhances formation of the Pol $\beta$-Ub conjugate. Thus, addition of SDS may represent a general approach to improve the conjugation efficiency of Ub to any protein of interest. Further experiments will clarify this aspect by utilizing conjugation partners other than Pol $\beta$.

In conclusion, two major methodical advances for the efficient generation of bioorthogonally linked, defined mono-ubiquitylated proteins have been made. Improved availability of such conjugates will offer new possibilities to study the diverse effects of ubiquitylation.

\section{Experimental section}

\subsection{Expression and purification of Ub G76Aha}

The gene coding for human Ub G76M was codon optimized for expression in E. coli and synthesized by GeneArt. Upon removal of the $5^{\prime}$ ATG codon, the cDNA was cloned into the pGEX2TK plasmid 
using BamHI and EcoRI, resulting in an open reading frame encoding an $\mathrm{N}$-terminal GST fusion to Ub. For recombinant expression, Met auxotrophic $E$. coli B834 (DE3) was transformed with this construct. Cells were cultured in NMM, ${ }^{50}$ containing $0.06 \mathrm{mM}$ Met for $14-18 \mathrm{~h}$ at $37^{\circ} \mathrm{C}$, until stationary phase was reached $\left(\mathrm{OD}_{600}=0.9\right.$ 1.0). Cells were harvested by centrifugation and resuspended in fresh NMM containing $0.5 \mathrm{mM}$ Aha corresponding to $10 \%$ of the initial culture volume. After incubation for $30 \mathrm{~min}$ at $37^{\circ} \mathrm{C}$, expression of the GST-Ub G76M fusion construct was induced with $1 \mathrm{mM}$ IPTG. After additional $4 \mathrm{~h}$, cells were harvested by centrifugation and resuspended in lysis buffer ( $1 \times$ PBS, $1 \%$ Triton X-100) and lysed by sonication. The lysate was clarified by centrifugation and the supernatant was loaded onto a GST-TrapFFTM column (GE HEALTHCARE). GST-Ub G76Aha was incubated with 5-20 U thrombin for $14 \mathrm{~h}$ at room temperature to cleave the fusion protein downstream of GST. Ub G76Aha was purified by washing the column with $1 \times$ PBS buffer. The purity of the resulting samples was judged by SDS-PAGE, the concentration of Ub was determined by absorption spectroscopy and samples were stored at $4{ }^{\circ} \mathrm{C}$.

\subsection{Effect of expression culture cell density on the yield of GST-Ub G76Aha}

E. coli B834 (DE3) harboring pGEX2TK_Ub G76Aha were cultured in NMM containing $0.06 \mathrm{mM}$ Met at $37^{\circ} \mathrm{C}$ for $14-18 \mathrm{~h}$ until stationary phase was reached. Different volumes of the culture $(100,50$ and $10 \mathrm{ml})$ were harvested by centrifugation. Each sample was resuspended in $10 \mathrm{ml}$ fresh NMM containing $0.5 \mathrm{mM}$ Aha, resulting in three expression cultures with different cell densities $\left(\mathrm{OD}_{600}=0.8,4.0\right.$ or 8.0$)$. After incubation for $30 \mathrm{~min}$ at $37^{\circ} \mathrm{C}$, expression of the GST-Ub G76M fusion construct was induced with $1 \mathrm{mM}$ IPTG and cells were incubated for additional $4 \mathrm{~h}$. Cells were harvested by centrifugation, resuspended in lysis buffer $(1 \times$ PBS, $1 \%$ Triton X-100) and lysed by sonication. Glutathione beads were added to clarified lysates and samples were gently agitated for $2 \mathrm{~h}$ at $4{ }^{\circ} \mathrm{C}$. The beads were washed 3 times with lysis buffer and finally supplemented with SDS-loading buffer. For all three expression cultures, equal sample volumes were analyzed by SDS-PAGE followed by Commassie blue staining.

\subsection{Expression and purification of Pol $\beta$ K61Plk}

The nucleotide sequence of human Pol $\beta$, modified with an Nterminal $\mathrm{His}_{6}$-tag and a small linker (TDP) between tag and polymerase, was optimized for expression in $E$. coli and synthesized by GeneArt. The Lys 61 codon of Pol $\beta$ was replaced by an amber codon, using site-directed mutagenesis. The resulting cDNA was inserted into the multiple cloning site of pET11a using HindIII and $\mathrm{XbaI}$. The vector additionally contained an expression cassette for the tRNA ${ }^{\text {Pyl }}$ gene in its backbone. ${ }^{36}$ For recombinant expression via amber codon suppression, E. coli BL21 (DE3) was co-transformed with this plasmid and the pRSFDuet- 1 vector, containing the PylRS gene from Methanosarcina barkeri. ${ }^{36}$ Cells were cultured in LB medium supplemented with the appropriate antibiotics at $37^{\circ} \mathrm{C}$. At an $\mathrm{OD}_{600}=0.3,1 \mathrm{mM}$ Plk was added and at $\mathrm{OD}_{600}=0.8$, expression was induced with $1 \mathrm{mM}$ IPTG. After $7 \mathrm{~h}$, cells were harvested, resuspended in lysis buffer $(20 \mathrm{mM}$ Tris- $\mathrm{HCl}, 500 \mathrm{mM} \mathrm{NaCl}$, and $\mathrm{pH} 7.9$ ) supplemented with $1 \mathrm{mM}$ PMSF and $5 \mathrm{mM}$ imidazole. Cell lysis was achieved by sonication. The lysate was clarified by centrifugation and $\mathrm{Ni}^{2+}$-IDA beads were added to the supernatant. After incubation for $1 \mathrm{~h}$ at $4{ }^{\circ} \mathrm{C}$, beads were washed with lysis buffer supplemented with increasing concentrations of imidazole $(10$, 15 , and $50 \mathrm{mM}$ ). Following elution of Pol $\beta$ with $250 \mathrm{mM}$ imidazole, dialysis against $20 \mathrm{mM}$ Tris- $\mathrm{HCl}, 50 \mathrm{mM} \mathrm{NaCl}, \mathrm{pH} 7.9$ was performed. Subsequently, the protein was loaded onto a HiTrap ${ }^{\mathrm{TM}}$ SP
HP column (GE HEALTHCARE) and eluted with a linear gradient from $50 \mathrm{mM}$ to $1 \mathrm{M} \mathrm{NaCl}$ over 15 column volumes. Fractions containing pure Pol $\beta$ were pooled, dialysed against $20 \mathrm{mM} \mathrm{NaAc}, \mathrm{pH}$ 5 and the protein concentration was determined by absorption spectroscopy. For storage, glycerol was added to a final concentration of $50 \%$ and samples were kept at $-20^{\circ} \mathrm{C}$.

\subsection{Click reaction}

Pol $\beta$ K61Plk at a final concentration of $5-10 \mu \mathrm{M}$ in $20 \mathrm{mM} \mathrm{NaAc}$ pH 5.0 was mixed with a five to 10 -fold molar excess of Ub G76Aha in $1 \times$ PBS. For the reaction, different amounts of either ascorbate or TCEP as reductant, TBTA and $\mathrm{CuSO}_{4}$ were added. Final concentrations of reductant and $\mathrm{CuSO}_{4}$ between 1 and $5 \mathrm{mM}$ were tested, with ratios ranging from $1: 1$ to $5: 1$. The amount of TBTA versus $\mathrm{CuSO}_{4}$ was varied between $0.01: 1$ and $0.1: 1$. In order to prevent $\mathrm{Cu}$-induced protein oxidation, reaction vessels were flushed multiple times with argon. After incubation at $4^{\circ} \mathrm{C}$ for $15 \mathrm{~min}$ up to $120 \mathrm{~min}$, reactions were stopped with a 10 -fold molar excess of EDTA over copper. Yield of product was analyzed by SDS-PAGE followed by Coomassie blue staining.

\subsection{Mass analysis}

The removal of the N-terminal Aha by thrombin cleavage was confirmed by mass analysis of the full-length protein by ESI-MS. The mass corresponding to full-length Pol $\beta$ K61Plk was determined by LC-ESI-MS. To confirm the linkage formed by click reaction, a trypsin in-gel digest was performed and the resulting fragments were analyzed by MALDI-MS.

The thrombin-cleaved Ub G76C was measured by ESI-MS at the 'Functional Genomics Center Zurich'. For MALDI-MS, samples were desalted via Pierce ${ }^{\circledR}$ C18 Tips (Thermo Scientific) and analyzed on a Bruker Microflex MALDI-TOF. LC-ESI-MS was performed on a Thermo LTQ Orbitrap Discovery equipped with an Eksigent 2D-nano HPLC for LC.

\section{Acknowledgments}

We gratefully acknowledge funding by DFG (SFB 969) and the Konstanz Research School Chemical Biology. We thank the Proteomics Facility Konstanz for assistance with mass spectrometry.

\section{References and notes}

1. Seet, B. T.; Dikic, I.; Zhou, M. M.; Pawson, T. Nat. Rev. Mol. Cell Biol. 2006, 7, 473.

2. Hershko, A.; Ciechanover, A. Annu. Rev. Biochem. 1998, 67, 425.

3. Haglund, K.; Dikic, I. EMBO J. 2005, 24, 3353.

4. Hoeller, D.; Hecker, C. M.; Dikic, I. Nat. Rev. Cancer 2006, 6, 776.

5. Sigismund, S.; Polo, S.; Di Fiore, P. P. Curr. Top. Microbiol. Immunol. 2004, 286, 149.

6. Peng. J.; Schwartz. D.; Elias, J. E.; Thoreen, C. C.: Cheng, D.; Marsischky, G.; Roelofs, J.: Finley, D.; Gygi, S. P. Nat. Biotechnol. 2003, 21, 921.

7. Li, W.; Ye, Y. Cell. Mol. Life Sci. 2008, 65, 2397.

7. Li, W.; Ye, Y. Cell. Mol. Life Sci. 2008, 65, 2397.
8. Hicke, L. Nat. Rev. Mol. Cell Biol. 2001, 2, 195.

9. Kerscher, O.; Felberbaum, R.; Hochstrasser, M. Annu. Rev. Cell Dev. Biol. 2006, 22. 159.

10. Welchman, R. L.; Gordon, C.; Mayer, R. J. Nat. Rev. Mol. Cell Biol. 2005, 6, 599.

11. Hoege, C.; Pfander, B.; Moldovan, G. L.; Pyrowolakis, G.; Jentsch, S. Nature 2002. $419,135$.

12. Kannouche, P. L.; Wing. J.; Lehmann, A. R. Mol. Cell 2004, 14, 491

13. Ulrich, H. D.; Jentsch. S. EMBO J. 2000, 19, 3388

14. Li, M.; Brooks, C. L.; Wu-Baer, F.; Chen, D.; Baer, R.; Gu, W. Science 2003, 302 , 1972.

15. Lohrum, M. A.; Woods, D. B.; Ludwig, R. L.; Balint, E.; Vousden, K. H. Mol. Cell. Biol. 2001, 21, 8521.

16. Pham, A. D.: Sauer, F. Science 2000, 289, 2357.

17. Terrell, J.; Shih, S.; Dunn, R.; Hicke, L. Mol. Cell 1998, 1, 193

18. Parsons, J. L.; Tait, P. S.; Finch, D.; Dianova, II; Edelmann, M. J.; Khoronenkova, S. V.; Kessler, B. M.; Sharma, R. A.; McKenna, W. G.; Dianov, G. L. EMBOJ. 2009, 28, 3207.

19. Bergink, S.: Jentsch. S. Nature 2009, 458, 461. 
20. Dianov, G. L.; Meisenberg, C.; Parsons, J. L. Biochemistry (Moscow) 2011, 76, 69. 21. Huang, T. T.; D’Andrea, A. D. Nat. Rev. Mol. Cell Biol. 2006, 7, 323.

22. Thomson, T. M.; Guerra-Rebollo, M. Biochem. Soc. Trans. 2010, 38, 116.

23. Pickart, C. M.; Fushman, D. Curr. Opin. Chem. Biol. 2004, 8,610

24. Pickart, C. M.; Raasi, S. Methods Enzymol. 2005, 399, 21.

25. Piotrowski, J.; Beal, R.; Hoffman, L.; Wilkinson, K. D.; Cohen, R. E.; Pickart, C. M. J. Biol. Chem. 1997, 272, 23712

26. Fekner, T.: Li. X.: Chan. M. K. ChemBioChem 2011, 12, 21.

27. Spasser, L.: Brik, A. Angew, Chem, Int. Ed. 2012, 51, 6840.

28. Strieter, E. R.; Korasick, D. A. ACS Chem. Biol. 2012, 7, 52.

29. McGinty, R. K.; Kim, J.; Chatterjee, C.; Roeder, R. G.; Muir, T. W. Nature 2008 $453,812$.

30. Kim. J.: Guermah, M.; McGinty. R. K.; Lee, J. S.; Tang. Z.; Milne, T. A.; Shilatifard A.; Muir, T. W.; Roeder, R. G. Cell 2009, 137, 459

31. Kumar, K. S.; Spasser, L.; Erlich, L. A.; Bavikar, S. N.; Brik, A. Angew. Chem., Int. Ed. 2010, 49, 9126 .

32. Shanmugham, A.: Fish, A.; Luna-Vargas, M. P. A.; Faesen, A. C.: Oualid, F. E.; Sixma, T. K.; Ovaa, H. J. Am. Chem. Soc. 2010, 132, 8834.

33. Jung. J. E.; Wollscheid, H. P.; Marquardt, A.; Manea, M.; Scheffner, M.; Przybylski, M. Bioconjug. Chem. 2009, 20,1152.

34. Chatterjee, C.; McGinty, R. K.; Fierz, B.; Muir, T. W. Nat. Chem. Biol. 2010, 6, 267.

35. Sommer, S.; Weikart, N. D.; Brockmeyer, A.; Janning, P.; Mootz, H. D. Angew. Chem., Int. Ed. 2011, 50, 9888.

36. Eger, S.; Scheffner, M.; Marx, A.; Rubini, M. J. Am. Chem. Soc. 2010, 132, 16337.

37. Weikart, N. D.; Mootz, H. D. ChemBioChem 2010, 11, 774.

38. Weikart, N. D.; Sommer, S.; Mootz, H. D. Chem. Commun. (Cambridge, (JK) 2012. $48,296$.

39. Eger, S.: Castrec, B.: Hubscher, U.: Scheffner, M.; Rubini, M.; Marx, A ChemBioChem 2011, 12, 2807.

40. Eger, S.; Scheffner, M.; Marx, A.; Rubini, M. Methods Mol. Biol, 2012, 832, 589.

41. Kolb, H. C.; Sharpless, K. B. Diug Discovery Today 2003, 8, 1128

42. Huisgen, R. Angew. Chem. 1963, 75, 604.

43. Tornøe, C. W.; Christensen, C.; Meldal, M. J. Org. Chem. 2002, 67, 3057.
44. Rostovtsev, V. V.; Green, L. G.; Fokin, V. V.; Sharpless, K. B. Angew, Chem. Int Fd 2002, 41, 2596.

45. Wang, Q.: Chan, T. R.; Hilgraf, R.; Folkin, V. V.; Sharpless, K. B.; Finn, M. G. J. Am Chem. Soc. 2003, $125,3192$.

46. Chan, T. R.; Hilgraf, R.; Sharpless, K. B.; Fokin, V. V. Org. Lett. 2004, 6. 2853.

47. Horne, W. S.; Stout, C. D.; Ghadiri, M. R. J. Am. Chem. Soc. 2003, 125, 9372.

48. Horne, W. S.; Yadav, M. K.; Stout, C. D.; Ghadiri, M. R. J. Am. Chem. Soc. 2004 126. 15366.

49. Brik, A.; Alexandratos, J.; Lin, Y.-C.; Elder, J. H.; Olson, A. J.: Wlodawer, A. Goodsell, D. S.; Wong, C.-H. ChemBioChem 2005, 6, 1167.

50. Budisa, N.; Steipe, B.; Demange, P.; Eckerskorn, C.; Kellermann, J.; Huber, R. Eu J. Biochem. 1995, 230, 788

51. Budisa, N.; Minks, C.; Alefelder, S.; Wenger, W.; Dong, F.; Moroder, L.; Huber, R. FASEB J. 1999, 13, 41.

52. Link, A. J.; Mock, M. L.; Tirrell, D. A Curr Opin. Biotechnol 2003, $14,603$.

53. Srinivasan, G.; James, C. M.; Krzycki, J. A. Science 2002, 296, 1459.

54. Hao. B.: Gong. W.: Ferguson, T. K.; James, C. M.: Krzycki, J. A.: Chan, M. K. Science 2002, 296, 1462

55. Polycarpo, C.: Ambrogelly, A.; Bérubé, A.; Winbush, S. A. M.: McCloskey, J. A Crain, P. F. Wood, J. L. Söll, D. Proc, Notl Acad, Sci. US. A 2004, 101, 12450.

56. Busso, C. S.; Iwakuma, T.; Izumi, T. Oncogene 2009, 28, 1616

57. Parsons, J. L.; Tait, P. S.; Finch, D.; Dianova, II; Allinson, S. L.; Dianov, G. L. Mol. Cell 2008, 29, 477

58. Ulrich, H. D. Curr. Biol. 2005, 15, R257.

59. Schwarz, S. E.; Rosa, J. L.; Scheffner, M. J. Biol. Chem. 1998, 273, 12148.

60. Liu, J.; Castaneda, C. A.; Wilkins, B. J.; Fushman, D.; Cropp. T. A. Bioorg. Med. Chem. Lett. 2010, 20. 5613.

61. Geoghegan, K. F.; Dixon, H. B.; Rosner, P. J.: Hoth, L. R.: Lanzetti, A. J.; Borzilleri, K. A.; Marr, E. S.; Pezzullo, L. H.; Martin, L. B.; LeMotte, P. K.; McColl, A. S.; Kamath, A. V.: Stroh, J. G. Anal. Biochem. 1999, 267, 169

62. Nielsen, M. M.; Andersen, K. K.; Westh, P.; Otzen, D. E. Biophys. J. 2007, 92, 3674 .

63. Otzen, D. E. Biophys. J. 2002, 83, 2219. 